\title{
A Scenarto for Estimating the charge on the Electron in \\ Terms of Planck's Constant and the Speed of Light
}

W. J. Swiatecki

\author{
Nuclear Science Diviston \\ Lawrence Berkeley Laboratory \\ Untversity of California \\ Berkeley, Callfornta 94720
}

May 1987

\section{DISCLAIMER}

\begin{abstract}
This report was prepared as an account of work sponsored by an agency of the United States Government. Neither the United States Government nor any agency thereof, nor any of their employees, makes any warranty, express or implied, or assumes any legal liability or responsibility for the accuracy, completeness, or usefulness of any information, apparatus, product, or process disclosed, or represents that its use would not infringe privately owned rights. Reference herein to any specific commercial product, process, or service by trade name, trademark, manufacturer, or otherwise does not necessarily constitute or imply its endorsement, recommendation, or favoring by the United States Covernment or any agency thereof. The views and opinions of authors expressed herein do not necessarily state or reflect those of the United States Government or any agency thereor.
\end{abstract}

This work was supported by the Director, Office of Energy Research, Diviston of Nuclear Physics of the offtce of High Energy and Nuclear Physirs of the U.S. Department of Energy under Contract OE-ACO3-76SFODU98.

\section{MASTER}

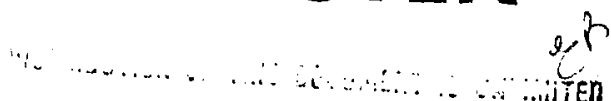


A Scenario for Estimating the Charge on the Electron in

Terms of Planck's Constant and the Speed of Light

\author{
W. J. Swiatecki \\ Nuclear Science Division \\ Lawrence Berkeley Laboratory \\ University of California \\ Berkeley, California 94720 , U.S.A.
}

May 1987

\title{
Abstract
}

The conjecture that the electron might be a soliton of a non-linearly generalized, charge-free electromagnetic field, together with an estimate of the strength of the hypothetical non-linearity (based on the standard QED calculation of the scattering of light by light) leads to an estimat:: of the soliton's charge, -e, such that $h c / e^{2}$ turns out to be equal to $45 \pi$ multiplied by a number of order one, whose precise value depends on the details of the soliton's radial form iactor.

\section{The electron as a hypothetical soliton}

Let us suppose that the electron is a stable (soliton) configuration of a quantized electromagnetic field $\vec{E}$, $\vec{B}$, the field obeying some non-linear equations that, in the weak-field limit, reduce to Maxwell's equations for pure radiation. There are supposed to be no intrinsic charges or currents in the theory. The hypothetical situation envisaged by this speculation is thus analogous to Skyrme's theory of schematic rucleons as solitons of a meson field. ") In both cases, there are no independent fermion fields. Only the bosons are there at the fundamental level. 
Is such a theory of electromagnetic or, more generally, of electro-weak phenomena possible? What is certain is that no one has so far constructed such a theory, but whether a theory of this type is in principle possible or impossible is less clear. We shall assume that the above question is to a certain extent an open one: it may all depend on the nature of the non-linear generalization of Maxwell's equations that one introduces. ${ }^{2)}$ It is certain, however, that all early attempts along the lines of the non-linear 'unitarian' theory of $M$. Born and $L$. Infeld ${ }^{3}$ ) would, sooner or later, have had to come up against the apparently insurmountable difficulty of making a spin $1 / 2$ fermion out of spin 1 bosons. Skyrme's discovery that, under certain conditions, a fermion can be made out of bosons, is crucial in changing the perspective on a hypothetical soliton interpretation of the electron.

For a soliton theory of the electron to be acceptable, it would have to reproduce quantitatively all the observable predictions of standard quantum eiectrodynamics, which assumes two coupled fields, photons and electrons, each of which separately is strictly linear. Using an 'inverted' point of view, the relation of the hypothetical non-linear theory of photons alone to standard QED could then be described as follows: the fundamental theory is supposed to be a non-linear theory of a single field, and standard QEO, together with its renormalization prescriptions, is merely a very accurate technique for linearizing the problem by the introduction of an auxiliary set of degrees of freedom, corresponding to phenomenological electrons (and positrons).

Granted that the above hypothesis may not be disprovable on theoretical grounds so long as the nature of the generalization of Maxwell's equations remains unspeciffed, is there anything useful to be learned from such a speculation? The purpose of this note is to suggest an affirmative answer 
and, in partfcular, to point to a scenario, based on the soliton hypothesis, for estimating $\mathrm{e}^{2}$ in terms of $\mathrm{hc}$. The leading idea here is very simple: in a pure photon theory, the fundamental constants at one's disposal are $h$ and $c$, and if, out of the photon field, one claims to be able to manufacture a soliton representing an electron, the effective strength (i.e. 'charge') of this electron must come out as some function of $h$ and $c$. On dimensional grounds the relation should be of the form $e^{2} \alpha \hbar c$, with the proportionality constant depending, presumably, on the strength of the hypothetical non-linearity. If we can find a way of somehow injecting an estimate of this strength, then an estimate of the proportionality factor might follow. Let us see how far we can get by basing ourselves on empirical facts as well as on theoretical results of standard quantum electrodynamics, but re-interpreted in terms of a soliton hypothesis.

\section{The soliton's energy density}

At large distances $r$ from the hypothetical soliton, we know empirically that it is characterized by an electric field $E(r)$, proportional to $1 / r^{2}$, or by an energy density $E^{2}(r) / 8 \pi$. (Whether the magnetic field due to the electron's magnetic moment should be included in an estimate of the energy density is discussed in section 5). The factor of proportionality between the electric field and $1 / r^{2}$ is conventionally called the electron's charge, -e. But, in the sprit of the soliton hypothesis, we are going to regard 'charge' as just a phenomenological, long-range characterization of the strength of the soliton configuration of the non-linearly generalized pure photon field.

Almost nothing is known about the appearance of the soliton's energy density at smaill distances, where the non-linearities that must be responsible 
$-4-$

for the soliton's stability become important and the asymptotic expression $E^{2}(r) / 8 \pi$ can no longer be valid. Two facts, however, one empirical and one theoretical, are relevant. First, we are entitled to assume that the space integral over the soliton's energy density is finite and equal to $\mathrm{mc}^{2}$, where $m$ is the electron's measured mass. Second, we know that the conventional quantized theory of the photon field coupled to the electron field may be interpreted in terms of an effective energy density of the photon field that begins to deviate from $E^{2} / 8 \pi$ according to the following (weak-field) perturbation formula ${ }^{4}$ ) (specialized here to the case where the magnetic field is absent):

$$
\text { effective energy density }=(1 / 8 \pi) E^{2}\left[1-\left(E / E_{s}\right)^{2}+\ldots\right] \text {, }
$$

where $E_{S}$, a characteristic field strength specifying the onset of the effective nonlinearity, is given by

$$
E_{s}=(45 \pi / h c)^{1 / 2}\left(m c^{2} / e\right)^{2}
$$

or

$$
E_{S}=\left(M c^{2}\right)^{2} /(h c)^{3 / 2}
$$

where

$$
M c^{2}=(45 \pi)^{1 / 4}\left(\hbar c / e^{2}\right)^{1 / 2} m c^{2},
$$


so that, numerically.

$$
M C^{2}=20.62679 \mathrm{MeV} .
$$

The non-linearity described by eq. (1) (when the magnetic field is included) accounts for the scattering of light by light, as predicted by standard QED. It is an effective non-linearity, resulting from eliminating from the theory of light the electron-positron degrees of freedom.

We shall now make use of the requirement that an acceptable soliton theory of the electron must agree with standard QEU predictions of observable phenomena, in particular as regards the prediction of the scattering of light by light. This means that eq. (1) should continue to hold in the hypothetical soliton theory, but now as an expression of the intrinsic non-linearity, rather than of an effective one. In this way, by an argument of 'correspondence' between the standard and the hypothetical theory, we obtain an estimate of the scale $E_{S}$ of the field strength at which non-linear effects would become important in the soliton theory.

Using this non-linearity scale $E_{S}$ we shall write the energy density of the soliton (no longer limited to the weak-field regime) as

$$
\text { energy density }=(1 / 8 \pi) E^{2} \Phi\left(E / E_{s}\right)
$$

where $\Phi$ is a dimensionless function of its dimensionless argument, with the property that 


$$
\Phi\left(E / E_{S}\right) \rightarrow 1 \text { for } E / E_{S} \ll 1 \text {. }
$$

The energy density is assumed to be integrable, and so the rest energy of the soliton may be written as

$$
\begin{aligned}
m c^{2} & =\int_{0}^{\infty} d r 4 \pi r^{2}(1 / 8 \pi) E^{2} \Phi\left(E / E_{s}\right) \\
& =E_{s}^{2} r_{s}^{3} N_{1}
\end{aligned}
$$

where

$$
N=(1 / 2) \int_{0}^{\infty} d x x^{2} n(x)
$$

and $n(x)$ stands for $\left(E / E_{S}\right)^{2} \Phi\left(E / E_{s}\right)$, a dimensionless energy-density function characteristic of the soliton. The dimensionless distance variable $x$ stands for $r / r_{s}$, where we have introduced the characteristic soliton size $r_{s}$ as that distance at which the unmodified electric field strength $e / r^{2}$ would have reached the value $E_{S}$. Thus $r_{S}$ is defined by

$$
e=E_{e} r_{s}^{2},
$$

and characterizes the distance below which deviations from an inverse fourth-power law for the soliton's energy density become important. The dimensionless quantity $N$ in eq. (8), a number of order one, is characteristic of the soliton's energy-density form factor. Since the conventional energy of 
a charged, hollow sphere with radius $r_{s}$ and surface field $E_{s}$ can be written as $E_{s}^{2} r_{s}^{3} / 2$, the physical meaning of the soliton form factor $N$ may be stated in words as the electron's rest energy $\mathrm{mc}^{2}$ in units of twice the rest energy of such a sphere.

3. The three equations whose solution gives $e^{2}$ in terms of $h c$

Squaring eq. (9), multiplying eq. (2) by $r_{s}^{2}$ and squaring, and multiplying eq. (7) by $r_{s}$, we obtain the following set of three equations for $e^{2}$ and for the two bracke ed unknowns $\left(E_{s}^{2} r_{s}^{4}\right)$ and $\left(m c^{2} r_{s}\right)$ :

$$
\begin{aligned}
& e^{2}=\left(E_{s}^{2} r_{s}^{4}\right) \\
& \left(E_{s}^{2} r_{s}^{4}\right)=45 \pi\left(m c^{2} r_{s}\right)^{4} / \hbar c e^{4} \\
& \left(m c^{2} r_{s}\right)=\left(E_{s}^{2} r_{s}^{4}\right) N .
\end{aligned}
$$

[q. (11) embodies the standard QED calculation of the field strength characterizing the theory's effective non-linearity, eq. (12) is the rest-energy expression and eq. (10) defines $r_{s}$ in terms of $E_{s}$ and $e$.

Solving for the three unknowns gives

$$
\begin{aligned}
& e^{2}=h c / 45 \pi N^{4} \\
& E_{s}^{2} r_{s}^{4}=h c / 45 \pi N^{4} \\
& m c^{2} r_{s}=h c / 45 \pi N^{3} .
\end{aligned}
$$


Eq. (13) gives for the value of e the expression

$$
e=(h c / 45 \pi)^{1 / 2} / N^{2}=0.0841 \sqrt{h C} / N^{2}
$$

and for the inverse of the fine structure constant $\alpha$

$$
\alpha^{-1} \equiv h c / e^{2}=45 \pi N^{4}=141.37 N^{4} \text {. }
$$

The value of $N$ that would reproduce the experimental value of the fine structure constant is

$$
N=(137.035963 / 45 \pi)^{1 / 4}=0.99224300 \text {. }
$$

Solving eq. (15) for $r_{s}$ one also finds

$$
r_{S}=(h / m c) / 45 \pi N^{3}=2.7314 \mathrm{fm} / \mathrm{N}^{3} \text {, }
$$

a quantity of the order of the classical electron radius $r_{0}\left(=e^{2} / m c^{2}=\right.$ $=2.82 \mathrm{fm})$. The field of a charge e evaluated at that distance is the predicted characteristic field strength $E_{S}$.

Note that the electron mass, $m$, has dropped out in the equation for $e$.

\section{Estimates of $\mathrm{N}$}

The integrand $I(x) \equiv x^{2} n(x)$ in eq. (8) tends to $1 / x^{2}$ for $x>>1$, when $n(x)$ tends to $1 / x^{4}$. For $x \leqslant 1$ the behavior of $I(x)$ is unknown, 
except that $I(x)$ is assumed to be integrable. If the crudest guess is made that

$$
I(x)= \begin{cases}1 / x^{2} & \text { for } x>1 \\ 1 & \text { for } x<1\end{cases}
$$

one finds $N=1$. This guess implies that for $x \rightarrow 0$ the energy density diverges (as $1 / x^{2}$ ). If one assumes instead that the energy density tends to a constant at the center of the soliton, $I(x)$ will start off parabolically at the origin. Interpolating by a cubic that ensures continuity of value and first dertvative at $x=1$ gives

$$
I(x)=5 x^{2}-4 x^{3} \quad \text { for } x<1
$$

and this leads to $N=5 / 6$. Interpolating with a fourth-order polynomial that ensures continuity of the second derivative as well, gives

$$
I(x)=15 x^{2}-24 x^{3}+10 x^{4}
$$

which happens to lead to $N=1$, once again.

For symmetry reasons one might prefer to interpolate with polynomials that are even in $x$. The resulting values of $N$ are $4 / 5,32 / 35$ or $64 / 63$ when continuity of value and of derivatives up to the first, second or third, respectively, is demanded.

The second-power sensitivity of e to $N$ in eq. (16) (fourth-power sensitivity of $\alpha$ ) makes it difficult to provide precise estimates of $e$ or $\alpha$ without more detailed information on the hypothetical soliton's 
energy-density form factor. This also raises the question of the possible contribution to these estimates coming from the electron's magnetic moment.

5. What about the magnetic energy?

The energy associated with the magnetic field of a classical dipole $\mu$, integrated from infinity down to a distance $r$, is given by $\mu^{2} / 3 r^{3}$. Since $\mu \approx \hbar e / 2 m c$ for an electron, this energy would be comparable to (in fact one sixth of) the electrostatic energy, if the integrations of the electric and magnetic energy densities were carried down to the compton wavelength $\mathrm{h} / \mathrm{mc}$. If the integrations were carried down to a distance of the order of the classical electron radius (the Compton wavelength divided by 137) the magnetic energy would overwhelm the electrostatic energy by a factor of $(137)^{2} / 6$, leading to a nonsensical estimate of the electron's rest energy and invalidating completely the arguments of the previous sections. However, the electron spin and the associated magnetic moment are, as is well known, dynamical phenomena, to which the satic estimate $/ 3 r^{3}$ does not apply - it is, in fact, completely misleading. In particular, as discussed by Weisskopf, ${ }^{5)}$ the 'Zitterbewegung' of the Dirac electron produces an alternating electric current (as well as a magnetic field responsibie for the magnetic moment). Weisskopf then derives the remarkable result that the effect of this alternating current is to cancel exactly the magnetic snergy, at least in the approximation where the polarization of the vacuum is neglected. With vacuum polarization and higher-order radiative corrections taken into account, both the electric and magnetic contributions become 
modified, but one is then in the regime where, according to the soliton speculation of the present paper, the hypothetical non-linearities are supposed to take over. Unfortunately, there is no unambiguous guidance from standard QED concerning the character of the energy density in this regime, since the spectacular achievements of renormalization theory do not include an estimate of the electron's mass or its energy-density profile. (The electron mass, which one might have hoped would be a precious empirical guide to the electron's constitution, in renormaliz:tion theory plays the undignified role of a convenient dumping ground for divergent integrals.)

In view of this state of things, we have not been able to go beyond an estimate according to which the electron's electrostatic field energy is taken at face value for $r>r_{5}$, whereas the magnetic energy is relegated to the shadowy regime of hypothetical non-"linearities.

\section{Suppose the electron had not been discovered}

Imagine a radiation dominated part of the world where the properties of the photon field had been studied in detail but, for some reason, the discrete nature of electricity had never been discovered. Thus we imagine that the energy density of the photon field, eq. (1), including the lowest-order non-linearity specified by eqs. (3) and (5), had been determined empirically (by the scattering of light by light). Since the electron had not been discovered, however, $h$ and $r$ but not $m$ and $e$ would appear in a local physicist's lit of natural constants. As a result, eq. (2), one of the three equations used in estimating $e$, would not be available. 
By contrast; imagine a different, particle dominated continent (where J.J. Thompson lived before Clerk Maxwel1), in which electrons and their electrostatic interactions were well known, but electromagnetic radiation had not been discovered or, at least, the relation of photons to electrostatics had not been realized.

Imagine now that communications between the two continents are established and a mathematical physicist in the radiation dominated continent receives a message about the existence of particles with mass $m=0.5110034$ $\mathrm{MeV} / \mathrm{c}^{2}$, which interact by inverse--square electric forces. The message fails, however, to say anything about the strength of the interaction. Our mathematical physicist wonders about the mysterious new particles and speculates that they might, perhaps, be solitons of the non-linear photon field with which he is familiar. He sets himself the task of estimating the strength of the particles' interactions, which the message failed to specify. Thus he imagines the particles to be stable, localized, soliton-like concentrations of the electric field $E$, with $E$ falling of $f$ as $1 / r^{2}$ for $r \gtrsim r_{s}$. He calls the product $E r^{2}$ (for large $r$ ) the strength or charge e of the soliton. He now estimates the self-energy of the soliton along the lines of section 2 and has then available the following three equations:

$$
\begin{aligned}
& m c^{2}=E_{s}^{2} r_{s}^{3} N \\
& E_{s}=\left(M c^{2}\right)^{2} /(h c)^{3 / 2} \\
& e=E_{s} r_{s}^{2},
\end{aligned}
$$


where $\mathrm{N} \approx 1, \mathrm{Mc}^{2}=20.62679 \mathrm{MeV}, \mathrm{mc}^{2}=0.5110034 \mathrm{MeV}$.

Solving these equations, he finds

$$
\begin{aligned}
e & =\sqrt{h c / N^{2 / 3}}(M / m)^{2 / 3} \\
& =0.08498222 \sqrt{\hbar C} / N^{2 / 3},
\end{aligned}
$$

or

$$
\mathrm{hc} / \mathrm{e}^{2}=(M / m)^{4 / 3} \mathrm{~N}^{4 / 3}=138.466219 \mathrm{~N}^{4 / 3}
$$

He also finds

$$
\begin{aligned}
r_{s} & =(\mathrm{h} / \mathrm{mc})(\mathrm{m} / \mathrm{M})^{4 / 3} / \mathrm{N}^{1 / 3} \\
& =2.7888 \mathrm{fm} / \mathrm{N}^{1 / 3} .
\end{aligned}
$$

Our mathematical physicist sends his estimated value of hc/e ${ }^{2}, e q .(27)$, to his distant colleagues, who are duly impressed. They send back a message saying that, in order to reproduce their empirical value of $k c / e^{2}$, his form factor $N$ would have to be

$$
N=(137.035963 / 138.466219)^{3 / 4}=0.99224300 \text {. }
$$


They all agree that, after this order-of-magnitude exercise, an interesting problem would be to explore the associated conjecture by trying to construct a concrete soliton model and check its properties against the available experimental data.

Note that if our mathematical physicist had been given the electron's charge but not its mass, he would have estimated the latter (using eqs. $(23)-(25))$ as

$$
m=M N\left(e^{2} / h c\right)^{3 / 4}=0.514998 N M e V / c^{2} .
$$

Finally, if he had been given neither the mass nor the charge of the particle, he would still have been able to estimate the ratio of the square of its mass to the cube of its charge as

$$
m^{2} / e^{3}=N^{2} M^{2} /(h c)^{3 / 2}
$$

7. Isn't $r_{s}$ too large and $E_{s}$ too small to be consistent with experiment? High energy electron scattering experiments set an upper limit on the electron's size that is three orders of magnitude below the soliton size $r_{s}$, as estimated above. Similarily, the electric field at the surface of a uranium nucleus is greater by a factor of about 13 than the electric field of an electron at its classical radius (our estimate of $E_{s}$ ), yet no evidence for non-linear deviations from Maxwell's equations has ever been found in relevant experiments. Is this a fatal blow to the soliton speculation? In 
this connection it is important to make a sharp distinction between non-linearties in the photon field that can be tolerated within the conventional two-field formulation of $Q E D$ and the non-linearities in a hypothetical one-field theory of photons alone. In the former case, the situation is clear-cut and the experimental constraints are, indeed, very tight. ${ }^{6)}$ In the latter case, a determination of the constraints would involve a discussion of the experimental data using the hypothetical soliton theory. This is, in the nature of things, not a well-defined proposition when the solitons, with their unspecified structure, are present. When solitons are not present, as in the interpretation of the scattering of light by light in the hypothetical theory (where the scattering is a direct result of the non-linearity of the photon field) then, of course, the magnitude of the non-linearity as described by $E_{S}$ is, by construction, precisely what is needed to achieve agreement with the standard predictions of QED (and with the presumed results of experiments, if these were available).

In more general situations, when solitons are present, one should bear in mind that the scattering of extended solitons of size $r_{s}$ might not at all be what one's intuition, based on the benavior of linear fields, would lead one to expect. In fact, the scattering of solitons is well known to exhirit anti-intuitive features. Thus, even classical, one-dimensional

Korteweg-deVries solitons') (e.g., idealized 'solitary waves' in a shallow canal) scatter strictly elastically, without any intrinsic excitations after the collision, despite their extended, non-rigid and non-quantal character. Similarly, little can be said with certainty about the behavior of a hypothetical soliton in the strong field of a heavy or super-heavy nucleus. (Some, at least, of the non-linarities that would show up in such situations 
would, presumably, be equivalent to what in conventional language is described as the effects of vacuum polarization and of higher-order radiative corrections.)

Recalling the 'inverted' point of view mentioned earlier, the above questions could be re-phrased as follows: how good an approximation to the non-linear soliton theory should the conventional two-field approach be expected to represent? Why does the mocking up of the solftons by means of a phenomenological electron-positron field work with such exquisite accuracy? It may well be that the soliton speculation will eventually be disproved in connection with these questions, but it would seem that the discussion will have to be carried on in the context of a soliton approach and, we believe, the answers are not clear at the present time.

\section{Summary and Conclusion}

Skyrme's discovery that under certain conditions one can make a fermion out of a boson, has removed a major obstacle against the conjecture that an electron might be a soliton of a non-linearly generalized photon field. In this note we have argued that: a) if one day some such soliton configuration is constructed, an estimate of $e^{2}$ in terms of hc will follow automatically, and b) if the resulting soliton theory were, in fact, found to be in quantitative agreement with the predictions of standard QED as regards the scattering of light by light, then $h \mathrm{c} / \mathrm{e}^{2}$ would come out to be $45 \pi$ multiplied iy a number of order one, a form factor reflecting the details of the soliton's structure. 
A final remark to forestall misunderstandings: this paper is not an estimate from first principles of the fine structure constant. It is rather a comment on the problem of the fine structure constant in the forni: "if one day an electron-like soliton is constructed ....then...". Its purpose is to draw attention to a conceivable scenario for calculating the fine structure constant and, perhaps, to stimulate further investigations along those lines. If an electron-like soliton is not found (and this is an only too easily conceivable alternative!) then the 'estimate' of $\mathrm{e}^{2} / \mathrm{hc}$ reported here will remain a mere numerical curiosity which is equivalent to one that, although not phrased in terms of such an estimate, had already been noted in the past (in refs. (4) and (6), for example).

\section{Acknowledgements}

I would like to thank J. Kalckar, F. Klinkhamer, J. Lindhard and J. Randrup for discussions.

This work was supported by the Director, Office of Energy Research, Division of Nuclear Physics of the Office of High Energy and Nuclear Physics of the U.S. Department of Energy under Contract DE-AC03-76SF00098. 


\section{References}

1. T. H. R. Skyrme, Nuc1. Phys, $\underline{31}$ (1962) 556; Proc. Roy. Soc. 262 (1961) 237; 260 (1961) 127; J. K. Perring and T. H. R. Skyrme, Nuc 1. Phys. 31 (1962) 550 .

2. For a collection of 76 papers on solitons, see C. Rebbi and G. Soliani "Solitons and Particles", World Scientific Publishing Co., 198?.

3. M. Born. Nature 132 (1933) 282; Proc. Roy. Soc. Al43 (1934) 410; M. Born and L. Infeld, Proc. Ray. Soc. Al44 (1934) 425; Al47 (1934) 522; Al50 (1935) 141.

4. H. Euler, Ann. Phys. 26 (1936) 398; V. B. Berestetskii, E. M. Lifshitz and L. P. Pitaevskii, "Quantum Eiectrodynamics" (Landau and Lifshitz Course on Theoretical Physics Vol. i, 2nd Edition), Pergamon press, 1982, especially paragraph 127, eq. (127.26).

5. V. F. Weisskopf, Phys. Rev. $\underline{56}$ (1939) 72.

6. J.D. Jackson, "Classical Electrodynamics ", 2nd ed., J.Wiley \& Sons, 1974. Sect. I.3; J. Rafelski, W. Greiner and L. P. Fulcher, Nuovn Cimento 13B (1973) 135. 